\title{
On Some Properties of the lbest Topology in Particle Swarm Optimization
}

\author{
Sayan Ghosh ${ }^{1}$, Debarati Kundu ${ }^{1}$, Kaushik Suresh ${ }^{1}$, Swagatam Das ${ }^{1}$, Ajith Abraham ${ }^{2}$, \\ Bijaya. K. Panigrahi ${ }^{3}$ and Václav Snášel ${ }^{4}$ \\ Department of Electronics and Telecommunication Engineering, Jadavpur University, Kolkata, India \\ ${ }^{2}$ Machine Intelligence Research Labs (MIR Labs), USA \\ ${ }^{3}$ Department of Electrical Engineering, Indian Institute of Technology, Delhi, India \\ ${ }^{3}$ Faculty of Electrical Engineering and Computer Science, VSB-Technical University of Ostrava, \\ Czech Republic \\ sayan88tito@gmail.com,kundu.debarati@gmail.com,kaushik_s1988@yahoo.com, \\ swagatamdas19@yahoo.co.in,ajithabraham@ieee.org,bkpanigrahi@ee.iitd.ac.in,vaclav.snasel@vsb.cz
}

\begin{abstract}
Particle Swarm Optimization (PSO) is arguably one of the most popular nature-inspired algorithms for real parameter optimization at present. The existing theoretical research on PSO is mostly based on the gbest (global best) particle topology, which usually is susceptible to false or premature convergence over multi-modal fitness landscapes. The present standard PSO (SPSO 2007) uses an lbest (local best) topology where a particle is stochastically attracted not towards the best position found in the entire swarm, but towards the best position found by any particle in its topological neighborhood. This paper presents a first step towards a probabilistic analysis of the lbest PSO with variable random neighborhood topology by addressing issues like inter-particle interaction and probabilities of selection based on particle ranks.
\end{abstract}

Keywords: Particle Swarm Optimization, Neighborhood Topologies, Swarm Intelligence, Global Optimization.

\section{Introduction}

The concept of particle swarm, although initially introduced for simulating human social behavior, has become very popular these days as an efficient means for intelligent search and optimization. The Particle Swarm Optimization (PSO) [1 - 5], as it is called now, does not require any gradient information of the function to be optimized uses only primitive mathematical operators and is conceptually very simple. Since its inception in 1995, PSO has attracted a great deal of attention of the researchers all over the globe resulting into nearly uncountable number of variants of the basic algorithm, theoretical and empirical investigations of the dynamics of the particles, parameter selection and control, and applications of the algorithm to a wide spectrum of real world problems from diverse field of science and engineering. For a comprehensive knowledge on the foundations, perspectives, applications of PSO [6 8].
The first stability analysis of the particle dynamics was due to Clerc and Kennedy [9]. They considered a deterministic approximation of the swarm dynamics by treating the random coefficients as constants, and studied stable and limit cyclic behavior of the dynamics for the settings of appropriate values to its parameters. A more generalized stability analysis of particle dynamics based on Lyapunov stability theorems was undertaken by Kadirkamanathan et al. [10]. Some other significant works in this direction can be found in [11-13, 22]. A majority of the research works including the above-mentioned theoretical studies on PSO are centered around the gbest PSO model, where a particle is attracted towards the best position found in the entire swarm. The gbest PSO, however, is susceptible to premature and/or false convergence over the multi-modal fitness landscapes [5, 14].

The current standard PSO (SPSO 2007) [14], obtainable from the Particle Swarm Central [15], uses an lbest topology where each particle is stochastically attracted to the best solution that any particle in their neighborhood has found.

In this work, we provide a simple probabilistic analysis of the information exchange among the particles in lbest PSO using the variable random topology model of SPSO 2007. The analysis provides important insights into the process of choosing the informants by a particle in variable random neighborhood. The analysis undertaken in this paper is the first of its kind and will provide a basis for the future theoretical investigation of the internal search mechanisms of lbest PSO.

\section{The Particle Swarm Optimization Algorithm}

\subsection{The Classical PSO}

The classical PSO starts with the random initialization of a population of candidate solutions (particles) over the fitness landscape. However, unlike other evolutionary computing techniques, PSO uses no direct recombination of genetic 
material between individuals during the search. Rather it works depending on the social behavior of the particles in the swarm. Therefore, it finds the global best solution by simply adjusting the trajectory of each individual towards its own best position and toward the best neighboring particle at each time-step (generation). In a $D$-dimensional search space, the position vector of the $i$-th particle is given by $\vec{X}_{i}=\left(x_{i, 1}, x_{i, 2}, \ldots \ldots ., x_{i, D}\right)$ and velocity of the $i$-th particle is given by $\vec{V}_{i}=\left(v_{i, 1}, v_{i, 2}, \ldots \ldots, v_{i, D}\right)$. Positions and velocities are adjusted and the objective function to be optimized $f\left(\vec{X}_{i}\right)$ is evaluated with the new coordinates at each timestep. The velocity and position update equations for the $d$-th dimension of the i-th particle in the swarm may be represented as:

$$
\begin{gathered}
v_{i, d, t}=\omega * v_{i, d, t-1}+C_{1} * \operatorname{rand}_{1} *\left(p_{i, d, t-1}^{l}-x_{i, d, t-1}\right) \\
+C_{2} * \operatorname{rand}_{2} *\left(p_{i, d, t-1}^{g}-x_{i, d, t-1}\right) \\
x_{i, d, t}=x_{i, d, t-1}+v_{i, d, t}
\end{gathered}
$$

where rand $_{1}$ and rand $_{2}$ are random positive numbers uniformly distributed in $(0,1)$ and are drawn anew for each dimension of each particle. $\vec{p}_{i}^{l}$ is the personal best solution found so far by an individual particle while $\vec{p}_{i}^{g}$ represents the best particle in a neighborhood of the $i$-th particle, for lbest PSO model. Note that in PSO, a neighborhood is defined for each individual particle as the subset of particles which it is able to communicate with. The gbest PSO may be regarded as a special case of the lbest model where the entire swarm acts as the neighborhood of any particle and nbest $_{i}$ simply becomes the globally best position found so far by all the particles in the population. In lbest PSO, if at any iteration, a particle is the best in its neighborhood, then the velocity update formula for this particle will be:

$$
v_{i, d, t}=\omega^{*} v_{i, d, t-1}+C_{1} * \operatorname{rand}_{1} *\left(p_{i, d}^{l}-x_{i, d, t-1}\right)
$$

The first term in the velocity updating formula is referred to as the 'cognitive part'. The last term of the same formula is interpreted as the 'social part', which represents how an individual particle is influenced by the other members of its society. $C_{1}$ and $C_{2}$ are called acceleration coefficients and they determine the relative influences of the cognitive and social parts on the velocity of the particle. The particle's velocity is clamped to a maximum value $\vec{V}_{\max }=\left[v_{\max , 1}, v_{\max , 2}, \ldots, v_{\max , D}\right]^{T} . \quad$ If $\quad$ in $d$-th dimension, $\left|v_{i, d}\right|$ exceeds $v_{\max , d}$ specified by the user, then the velocity of that dimension is assigned to $\operatorname{sign}\left(v_{i, d}\right) * v_{\max , d}$, where $\operatorname{sign}(x)$ is the triple-valued signum function.

\subsection{Topological variants of the classical PSO}

The basic PSO algorithm used in most of the existing papers implicitly uses a fully connected neighborhood topology (or gbest). Every particle is a neighbor of every other particle. Hence all particles are stochastically attracted towards the best solution found so far by any member of the swarm. Here each particle has access to the information of all other members in the community.

However, local neighborhood models (or lbest) have also been proposed for PSO long ago, where each particle has access to the information corresponding to its immediate neighbors, according to a certain swarm topology. The two most common topologies are the ring topology, in which each particle is connected with two neighbors and the wheel topology (typical for highly centralized business organizations), in which the individuals are isolated from one another and all the information is communicated to a focal individual. Kennedy and Mendes [17 - 19] evaluated a number of topologies presented as well as the case of random neighbors. Their research $[18,20]$ suggest that the gbest version converges fast but can be trapped in a local optimum very often, while the lbest network has more chances to find an optimal solution, although with slower convergence. The current standard PSO (SPSO'07 [16]) uses an lbest network with variable random neighborhood model and our present analysis will mostly be based on this model only. It is described in more details in the next subsection.

\subsection{The Variable Random Topology}

The variable neighborhood topology is described in Maurice Clerc's book on PSO [5] and it can be seen now as a particular case of the stochastic star of the work of Miranda et al. [21]. In this topology, there is no centralized concept of a global best. The particles select each other as informants, and out of these informants, one particle may be selected as the selecting particle's global best. The topology is highly dependent on a threshold probability $p$, which is constant for all particles in the swarm. Each particle assigns a uniformly distributed random value (between 0 and 1) to every other particle in the swarm. Then it checks how many of these particles have values less than the threshold $p$.

The particles having values less than $p$ are chosen as informants, implying that the selecting particle will attempt to select its global best from these particles. The best particle among the informants is chosen as the global best for the selecting particle. If the particle's own fitness is better than the best informant, the particle simply takes its own locally best position into account. 


\section{The Analytical Treatment}

Without the loss of generality, in the analysis that follows, we assume that the particles are arranged in an ascending order of their locally best fitness. From now on, when we refer to the $i$-th particle, we mean the $i$-th ranked particle. In the following theorems we shall derive the probabilities that the $i$-th particle selects the $j$-th particle i.e. the $j$-th ranked particle is selected as the globally best position by the $i$-th ranked particle. We shall show that a particle cannot select particles inferior to it.

Theorem 1: If $P_{i j}$ denotes the probability that the $i$-th ranked particle selects the $j$-th ranked particle as its global best where $i<j$ then $P_{i j}=0$.

Proof: The $i$-th particle compares its own fitness with the best fitness of the $k$-selected informants. If the best particle among $k$ members (here, the $j$-th particle) is worse than the fitness of the $i$-th particle then it cannot be selected. Thus the probability that the $i$-th ranked particle selects the $j$-th ranked particle as its global best becomes zero.

Lemma 1: If $n$ denotes the swarm size then the probability that the $i$-th ranked particle chooses itself (i.e. uses equation (3)) when the number of chosen informants is $k$, is given by

$$
P_{i i, k}=\frac{{ }^{n-i} C_{k}}{{ }^{n-1} C_{k}}
$$

Proof: The $i$-th particle chooses itself if it cannot find a particle superior to it among the chosen $k$ members. Thus the chosen $k$ particles consist only of particles inferior to it. Since there are $n-i$ particles inferior to it, the number of such possible combinations is ${ }^{n-i} C_{k}$. The total number of all possible combinations is given by ${ }^{n-1} C_{k}$. Hence the probability that the particle chooses itself is given by $P_{i i, k}=\frac{{ }^{n-i} C_{k}}{{ }^{n-1} C_{k}}$.

Lemma 2: If $P_{i j, k}$ denotes the probability that the $i$-th ranked particle selects the $j$-th ranked particle as its global best where $i>j$ and exactly $k$ informants are chosen, then

$$
P_{i j, k}=\frac{{ }^{n-1-j} C_{k-1}}{{ }^{n-1} C_{k}} .
$$

Proof: The $j$-th particle can be selected only if it is superior to all other particles from the chosen $k$ particles. There are $n-j$ particles inferior to the $j$-th particle and there are $n-j-1$ particles inferior when we exclude the selecting particle itself. We are effectively selecting $k-1$ particles, since the $j$ - th particle is already present among the chosen $k$ particles. The total number of such combinations is given by ${ }^{n-1-j} C_{k-1}$. The total number of all possible combinations is given by ${ }^{n-1} C_{k}$. The probability is hence

$$
P_{i j, k}=\frac{{ }^{n-1-j} C_{k-1}}{{ }^{n-1} C_{k}} .
$$

(Proved)

Two important observations follow. First, when the $j$-th ranked particle is selected by inferior particles, the selection probability is the same for all particles inferior to the $j$-th particle. The result of lemma 3 shows us that $P_{i j, k}=\frac{{ }^{n-1-j} C_{k-1}}{{ }^{n-1} C_{k}}$ is dependent only on $j, n$, and $k$.

In the following theorems, we find the respective probabilities that the $i$ th particle follows the $j$ th particle's locally best position, and that it follows its own locally best position. The results depend, to a large extent on the value of $p$, the probability with which each particle is selected as an informant.

Theorem 2: The probability that the $i$-th particle follows the locally best position of the $j$-th particle is given by

$$
P_{i j}=p \cdot(1-p)^{j-1} \text {. }
$$

Proof: We first derive the probability with which exactly $k$ informants are selected. Out of $n-1$ particles, there are ${ }^{n-1} C_{k}$ ways in which $k$ particles can be selected as informants. For each combination, the probability that $k$ particles are chosen and $n-1-k$ particles are not chosen as informants is given by $p^{k}(1-p)^{n-1-k}$. Hence the total probability that exactly $k$ particles are chosen as informants is given by $P_{k}={ }^{n-1} C_{k} p^{k}(1-p)^{n-1-k}$. When exactly $k$ informants are chosen, the probability that the $i$ th particle follows the locally best position of the $j$ th particle is given by $P_{i j k}=\frac{{ }^{n-1-j} C_{k-1}}{{ }^{n-1} C_{k}} \quad$ (from lemma 2).

We can find the probability that the $i$-th particle follows the $j$-th particle by summing over the entire range of $k$ from 0 to $n-1$ as follows:

$$
\begin{aligned}
P_{i j} & =\sum_{k=0}^{k=n-1} P_{i j k} \cdot P_{k} \\
& =\sum_{\mathrm{k}=0}^{\mathrm{k}=n-1}{ }^{n-1} C_{k} p^{k}(1-p)^{n-1-k} \frac{{ }^{n-1-j} C_{k-1}}{{ }^{n-1} C_{k}} \\
& =\sum_{\mathrm{k}=0}^{\mathrm{k}=\mathrm{n}-1}{ }^{n-1-j} C_{k-1} p^{k}(1-p)^{n-1-k}
\end{aligned}
$$


We substitute $\lambda=k-1$ in the above expression to obtain:

$$
\begin{aligned}
P_{i j} & =\sum_{\lambda=0}^{\lambda=n-2}{ }^{n-1-j} C_{\lambda} p^{\lambda+1}(1-p)^{n-\lambda-2} \\
& =p(1-p)^{j-1} \sum_{\lambda=0}^{\lambda=n-2}{ }^{n-1-j} C_{\lambda} p^{\lambda}(1-p)^{n-1-j-\lambda}
\end{aligned}
$$

The lower limit of $k$ is zero, and so the lower limit of $\lambda$ should be -1 . However the value of ${ }^{n-1-j} C_{\lambda}$ becomes zero for $\lambda=-1$, so we neglect the lower limit, and begin our summation from $\lambda=0$. Again, $j$ is a rank, so the inequality $1 \leq j \leq n$ holds. Hence $n-1-j \leq n-2$. Further ${ }^{n-1-j} C_{\lambda}=0$ for $\lambda>n-1-j$. Thus we can shift the upper limit of summation to $\lambda=n-1-j$. The expression for $P_{i j}$ is now given by:

$$
\begin{aligned}
P_{i j} & =p(1-p)^{j-1} \sum_{\lambda=0}^{\lambda=n-1-j}{ }^{n-1-j} C_{\lambda} p^{\lambda}(1-p)^{n-1-j-\lambda} \\
& =p(1-p)^{j-1}
\end{aligned}
$$

We arrive at the final expression through application of the binomial theorem.

Theorem 3: The probability that the $i$ th particle follows its own locally best position is given by

$$
P_{i i}=(1-p)^{i-1} \text {. }
$$

Proof: Proceeding in a similar manner to the proof of Theorem 2, we first derive the probability that exactly $k$ informants are selected. Out of $n-1$ particles, there are ${ }^{n-1} C_{k}$ ways in which $k$ particles can be selected as informants. For each combination, the probability that $k$ particles are chosen and $n-l-k$ particles are not chosen as informants is given by $p^{k}(1-p)^{n-1-k}$. Hence the total probability that exactly $k$ particles are chosen as informants is given by $P_{k}={ }^{n-1} C_{k} p^{k}(1-p)^{n-1-k}$. When exactly $k$ informants are chosen, the probability that the $i$ th particle follows its own locally best position is given by $P_{i i, k}=\frac{{ }^{n-i} C_{k}}{{ }^{n-1} C_{k}}$.

We can find the probability that the $i$ th particle follows itself by summing over the entire range of $k$ from 0 to $n-1$ as follows:

$$
\begin{aligned}
P_{i i} & =\sum_{k=0}^{k=n-1} P_{k} \cdot P_{i i, k} \\
& =\sum_{\mathrm{k}=0}^{k=n-1}{ }^{n-1} C_{k} p^{k}(1-p)^{n-1-k} \frac{{ }^{n-i} C_{k}}{n-1} C_{k} \\
& =\sum_{k=0}^{k=n-1}{ }^{n-i} C_{k} p^{k}(1-p)^{n-1-k} \\
& =(1-p)^{i-1} \sum_{k=0}^{k=n-1}{ }^{n-i} C_{k} p^{k}(1-p)^{n-i-k} \\
& =(1-p)^{i-1} \sum_{k=0}^{k=n-i}{ }^{n-i} C_{k} p^{k}(1-p)^{n-i-k} \\
& =(1-p)^{i-1}
\end{aligned}
$$

We have shifted the upper limit of $\lambda$ to $n-i$ in a manner similar to the proof of Theorem 2. Here also, we use the binomial theorem to arrive at the final expression.

The results of the theorem are highly dependent on the value of $p$. When $p=1$, the algorithm corresponds to the classical PSO, in which every particle of the swarm (excluding itself) is chosen as an informant for selection of the global best. The probability $P_{i j}$ evaluates to 0 when $j \neq 1$ and it evaluates to 1 when $j=1$. Thus all particles of the swarm follow the globally best position. The probability $P_{i i}=0$ when $i \neq 1$ which implies that all particles except the globally best particle cannot use equation (3) for velocity update. When $p=0$, the particles cease to interact with one another, with every particle following its own locally best position. The plots in Figure 1 show the probabilities $P_{i i}$ and $P_{i j}$ as functions of $p$.

\section{Conclusions}

This work attempts to capture an inner view of the particle interaction in the variable random topology of an lbest PSO by deriving the probabilities with which the particles exchange information among themselves. Since the particles choose informants on basis of their locally best fitness values, we have analyzed their interaction by assigning a fitness-based rank to each of them. We have also shown that the classical topology is a special case of the variable random topology of the lbest PSO when $p=1$. 


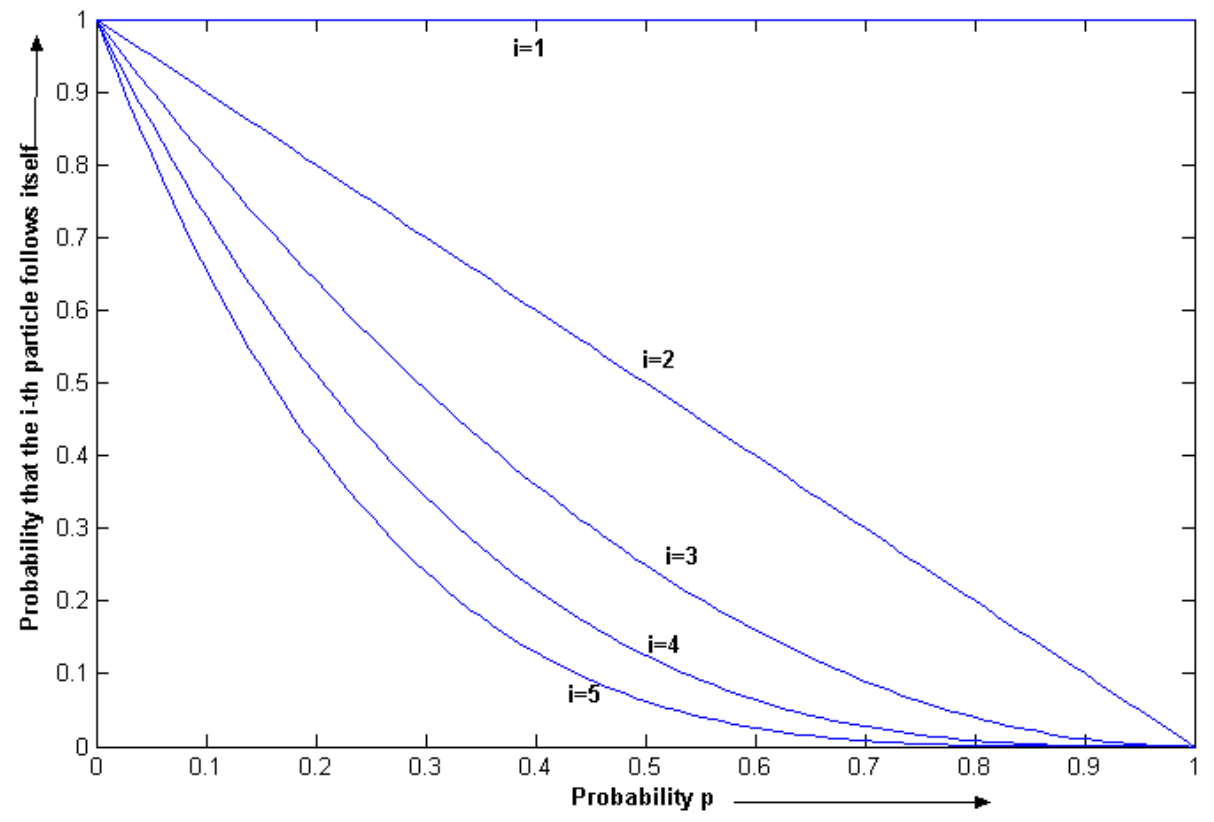

(a)

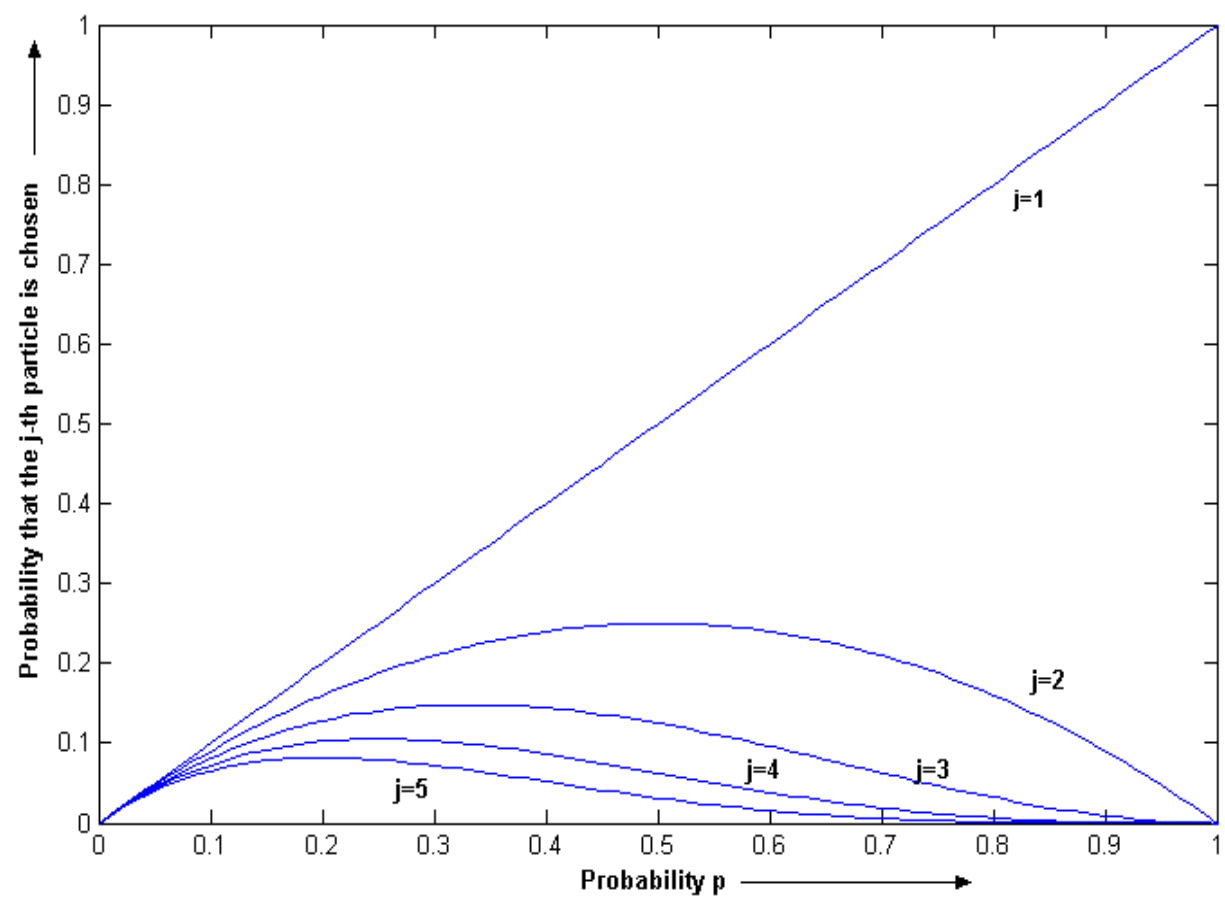

(b)

Figure 1. (a) Variation of $P_{i i}$ with $p$ for different values of $I$ and (b) Variation of $P_{i j}$ with $p$ for different values of $j$ 


\section{References}

[1] J. Kennedy and R. C. Eberhart: "Particle swarm optimization", In Proceedings of IEEE International conference on Neural Networks. 1942-1948, 1995.

[2] R. C. Eberhart and J. Kennedy, "A new optimizer using particle swarm theory," in Proc. $6^{\text {th }}$ Int. Symp. Micromachine Human Sci., vol. 1, Mar. 1995, pp. 3943

[3] J. Kennedy, R. C. Eberhart, and Y. Shi, Swarm Intelligence, Morgan Kaufmann, San Francisco, CA, 2001

[4] A. P. Engelbrecht, Fundamentals of Computational Swarm Intelligence, John Wiley \& Sons, 2006.

[5] M. Clerc, Particle Swarm Optimization, ISTE Publications, 2008.

[6] Y. del Valle, G. K. Venayagamoorthy, S. Mohagheghi, J. C. Hernandez, and R. G. Harley, "Particle Swarm Optimization: Basic Concepts, Variants and Applications in Power Systems", IEEE Transactions on Evolutionary Computation, Vol. 12, Issue 2, pp. 171 195, 2008.

[7] A. Banks, J. Vincent, and C. Anyakoha, A review of particle swarm optimization. Part I: background and development, Natural Computing: an international journal 6, 4, pp. 467-484, 2007.

[8] A. Banks, J. Vincent, and C. Anyakoha, A review of particle swarm optimization. Part II: hybridisation, combinatorial, multicriteria and constrained optimization, and indicative applications, Natural Computing: an international journal 7, 2, pp.109-124, 2008.

[9] M. Clerc and J. Kennedy, "The particle swarmexplosion, stability and convergence in a multidimensional complex space," IEEE Transactions on Evolutionary Computation, vol.6, no.2, pp. 58-73, Feb. 2002.

[10] V. Kadirkamanathan, K. Selvarajah, and P. J. Fleming, "Stability analysis of the particle dynamics in particle swarm optimizer," IEEE Transactions on Evolutionary Computation, vol.10, no.3, pp.245-255, Jun. 2006.

[11] I. C. Trelea, "The particle swarm optimization algorithm: convergence analysis and parameter selection," Inf. Process. Letters. vol. 85, pp. 317-325, Mar. 2003.

[12] N. R. Samal, A. Konar, S. Das and A. Abraham, "A closed loop stability analysis and parameter selection of the particle swarm optimization dynamics for faster convergence," in Proc. Congr. Evolu. Compu.(CEC 2007), Singapore, pp. 1769-1776, 2007.

[13] F. Van den Bergh, An analysis of particle swarm optimizers, Ph.D. dissertation, Univ. Pretoria, South Africa, 2002.

[14] D. Bratton and J. Kennedy, Defining a standard for particle swarm optimization, In IEEE swarm intelligence symposium, 2007.
[15] D. Bratton and T. Blackwell, Understanding particle swarms through simplification: a study of recombinant PSO. In Proceedings of the 2007 GECCO Conference Companion on Genetic and Evolutionary Computation (London, United Kingdom, July 07 - 11, 2007). GECCO '07. ACM, New York, NY, 2621-2628, 2007.

[16] www.particleswarm.info/

[17] J. Kennedy, "Small Worlds and Mega-Minds: Effects of Neighborhood Topology on Particle Swarm Performance", Proceedings of the 1999 Congress of Evolutionary Computation, vol. 3, IEEE Press, (1999): 1931-1938.

[18] J. Kennedy and R. Mendes, "Population structure and particle swarm performance", Proceedings of the Congress on Evolutionary Computation (CEC 2002), pages 1671-1676, Honolulu, HI, USA, 2002.

[19] R. Mendes, J. Kennedy and J. Neves, "The fully informed particle swarm: simpler, maybe better," IEEE Transactions on Evolutionary Computation, Vol. 8, no. 3, pp. 204-210, 2004.

[20] R. Mendes, J. Kennedy and J. Neves, "Avoiding the pitfalls of local optima: how topologies can save the day", Proceedings of the 12th Conference Intelligent Systems Application to Power Systems (ISAP2003), IEEE Computer Society, 2003.

[21] V. Miranda, H. Keko, and A. J. Duque, "Stochastic Star Communication Topology in Evolutionary Particle Swarms (EPSO)", International Journal of Computational Intelligent Research, 2008.

[22] H. Liu, A. Abraham and M. Clerc, Chaotic Dynamic Characteristics in Swarm Intelligence, Applied Soft Computing Journal, Elsevier Science, Volume 7, Issue 3, pp. 1019-1026, 2007. 